\title{
A COMPARATIVE STUDY OF GRID-IRON TECHNIQUE AND LICHTENSTEIN TECHNIQUE IN INGUINAL HERNIA REPAIR
}

\author{
Rasbihari Hembram¹, Bidyut Kumar Biswas ${ }^{2}$
}

${ }^{1}$ Assistant Professor, Department of General Surgery, College of Medicine and JNM Hospital, Kalyani. ${ }^{2}$ RMO/Clinical Tutor, Department of General Surgery, College of Medicine and JNM Hospital, Kalyani. \begin{abstract}
BACKGROUND

The advances in groin hernia repair in the century following Bassini have shared the primary goal of reducing long-term hernia recurrence rates. The surgeon must have a comprehensive understanding of the anatomy of the groin to properly select and utilise various options for hernia repair.

The aim of our study was to compare the Grid-Iron technique and the Lichtenstein method of open hernia repair in terms of operative time, post-operative pain, post-operative complications, hospital stay, return to normal activity and early recurrence rate.
\end{abstract}

\section{MATERIALS AND METHODS}

This prospective randomised comparative study period was conducted from August 2015 to July 2017 with a sample size of 60 cases in Kalyani Medical College and included patients who reported to outpatient department. Patients were randomised into two groups, one group underwent repair of inguinal hernia by Grid-Iron technique and the other by Lichtenstein technique.

\section{RESULTS}

Those who underwent Grid-Iron technique of repair had less intensity post-operative pain, shorter duration of hospital stay and returned to work earlier; whereas those who had undergone Lichtenstein repair had less post-operative complications and had no recurrence unlike in other group which had two recurrences.

\section{CONCLUSION}

Lichtenstein repair of inguinal hernia is a time-tested method of treatment of inguinal hernia. It is associated with the lowest rate of recurrence among the various methods of repair. Being tension-free it allows the patient to early return to previous state of activity, whereas Grid-Iron technique is considered a more physiological repair having all the advantages of Minimal Access Surgery like of quicker return to work, less post-operative pain and a better cosmetic result.

\section{KEYWORDS}

Inguinal Hernia, Grid-Iron Technique, Lichtenstein Technique, Hernia Repair.

HOW TO CITE THIS ARTICLE: Hembram R, Biswas BK. A comparative study of grid-iron technique and Lichtenstein technique in inguinal hernia repair. J. Evolution Med. Dent. Sci. 2017;6(91):6501-6509, DOI: 10.14260/jemds/2017/1412

\section{BACKGROUND}

Hernias of the abdominal wall are the most common conditions requiring surgery. The groin is one of the natural weak areas of the abdominal wall and is the most common site for abdominal herniation. Both sexes of all ages are affected, but men are more likely to have groin hernia than women.[1]

Inguinal hernias can be congenital or acquired, and in both a family history of groin hernia is strongly positive. Most hernias of the groin therefore may be said to be transmitted genetically. Indirect inguinal hernias are congenital and result from patent processus vaginalis with which the patient is born. A patent processus vaginalis is found in $80 \%$ of newborns and in $50 \%$ of one-year olds. Closure continues until the age of two years. The incidence of patent processus

'Financial or Other Competing Interest': None.

Submission 09-10-2017, Peer Review 10-11-2017,

Acceptance 18-11-2017, Published 27-11-2017.

Corresponding Author:

Dr. Bidyut Kumar Biswas,

RMO/Clinical Tutor,

Department of General Surgery,

College of Medicine and JNM Hospital,

Kalyani, Nadia-741235.

E-mail: pratheek.n2012@gmail.com

DOI: $10.14260 /$ jemds $/ 2017 / 1412$

\section{(c) $(1)$}

vaginalis in adult is $20 \%$. However, having the potential for hernia does not mean that a hernia will develop. Other factors must be present to cause failure of transversalis fascia to retain the visceral sac in myopectineal orifice.[2]

The erect stance of human beings in contrast to that of four legged animals promotes herniation by stretching and exposing the groin, and when a hernia is present permitting the dependent intestine to drop down into hernia sac.[3]

Muscle deficiency contributes to herniation. Congenital or acquired deficiencies of the internal oblique muscle in the groin exposes the deep ring and the floor of the inguinal canal to the destructive effects of the intra-abdominal pressure.

Destruction of the connective tissue resulting from the physical stress of the intra-abdominal pressure, smoking, aging, connective tissue disorder and systemic illnesses reduce the strength of the transversus abdominis fascia. Fracture of the elastic fibres and alterations in structure, quantity and metabolism of collagen have been demonstrated in connective tissue structures of the groin in patients with hernias. [4]

Other factors may also have an effect in some cases. Abdominal distention and chronic increase in intraabdominal pressure from ascites and peritoneal dialysis may damage the myopectineal orifice and cause a patent processus vaginalis to dilate. Fracture deformities of the pelvis and denervation of shutter mechanism following a low 
cosmetic appendectomy incision are well known, but uncommon causes of inguinal hernia. Inguinal hernias of all types occur equally in sedentary and physically active men. Vigorous physical activity per se is not the cause of inguinal hernia, although strenuous effort may aggravate predisposing factors and precipitate herniation.

Bassini[2] revolutionised the surgical repair of the groin hernia with his novel anatomical dissection and low recurrence rates. He first performed his operation in 1884 and published his initial outcomes in 1889. Bassini reported $100 \%$ follow-up of patients over a 5 -year period with just 5 recurrences in over 250 patients. This rate of recurrence was unheard of at the time and marked a distinct turning point in the evolution of herniorrhaphy. Bassini's repair emphasises both the high ligation of the hernia sac in the internal ring as well as suture reinforcement of the posterior inguinal canal. The operation utilises a deep and superficial closure of the inguinal canal.

The advances in groin hernia repair in the century following Bassini have shared the primary goal of reducing long-term hernia recurrence rates. To this end, efforts have been directed at developing a repair that imparts the least tension on the tissues that are brought together to repair the hernia defect.

The modern synthetic patch, made of a plastic monofilament polymer (polyethylene) was introduced by Usher in 1958. Lichtenstein, who developed a sutureless hernia repair using a plastic mesh patch placed across the inguinal floor further popularised this technique.

In the search for a technical means to reduce recurrence, emphasis was also placed on a meticulous dissection that would avoid placement of a prosthetic mesh. The most popular version was the Shouldice technique, initially introduced in 1958, and in essence a modification of the Bassini operation.[5] This technique involves meticulous dissection of the entire inguinal floor and closure of the inguinal canal in four layers. The transversalis fascial layer itself is closed in two layers as opposed to the single layer of interrupted suture advocated by Bassini. While the operation can be technically challenging to the beginner, it has been associated with excellent long-term outcomes and low recurrence rates.

Today, laparoscopic techniques have been validated as safe and effective in the treatment of groin hernias and have become the common place. The laparoscopic approaches were initially developed in the early 1990s, as laparoscopic techniques diffused throughout other specialties of general surgery.

The surgeon must have a comprehensive understanding of the anatomy of the groin to properly select and utilise various options for hernia repair.

\footnotetext{
Aims and Objectives Repair in Terms of-

1. Operative time.

2. Post-operative pain.

3. Post-operative complications

4. Hospital stay.

5. Return to normal activity.

6. Early recurrence rate.
}

The Aim of our Study was to compare the Grid-Iron Technique and the Lichtenstein Method of Open Hernia

\section{MATERIALS AND METHODS}

This prospective randomised comparative study period was conducted from August 2015 to July 2017 with a sample size of 60 cases in Kalyani Medical College and included patients who reported in outpatient department having diagnosed with inguinal hernia. Patients were included without a bias on a serial basis. Patients were randomised into two groups, one group underwent repair of inguinal hernia by grid-iron technique and other by Lichtenstein technique with 30 in each group. Randomisation was done by using the SAS software.

Both sexes with unilateral, primary or first recurrence inguinal hernia were included in the study.

Patients were excluded if they were medically unfit for surgery. Also, patients with previous lower or paramedian incision, those with more than one recurrence, coagulation disorder, those with irreducible hernia or needed emergency surgery for obstruction, strangulation, peritonitis, perforation and patients with systemic or local infection were excluded. Pregnant women were also excluded.

The qualifying patients were informed of the risk and benefits of each operation and were asked to sign a detailed informed consent in their native language. Ethical committee clearance was obtained.

\section{Technique}

All the patients underwent standardised repair by residents with an attending surgeon experienced in both the methods. All repairs involved the use of mesh under spinal anaesthesia. Recurrent hernias were repaired by the same standardised method as for primary hernias. All the patients were given standardised post-operative instructions that did not restrict their activities unless the activities caused pain.

\section{Lichtenstein Technique[6]}

A transverse skin incision is made down to the external oblique. The external oblique is divided. After that the direct sacs are inverted and imbricated with absorbable suture; indirect sacs are dissected high and then inverted or ligated. The posterior wall is then covered with $6 \times 10 \mathrm{~cm}$ piece of mesh with longitudinal slit laterally to give one-third lower leaf and two-third upper leaf distribution to the mesh; 2 to 3 $\mathrm{cm}$ of overlap is obtained at the pubic tubercle and the inferior border of the mesh is sutured to Poupart's ligament with loose continuous suture. The superior edge is attached to the internal oblique and conjoint tendon with interrupted sutures and one or two sutures are used where the tails of the mesh cross lateral to the cord, attaching them to the inguinal ligament and creating snug fit around the cord. The wound is then closed in usual fashion.

\section{Grid-Iron Technique[7,8]}

In this technique, the approach of MPO for mesh insertion will be lateral. After the $3-4 \mathrm{~cm}$ skin incision is made, sharp dissection is performed down to the aponeurosis of the external oblique muscle. The aponeurosis is divided parallel to its fibres after which the internal oblique muscle can be identified. The ilioinguinal nerve crossing on the caudal side must be identified and avoided. Cranial to this nerve, the internal oblique and transversalis muscles are also opened through their fibres. Until this part of the operation we must stay lateral, do not cross the fascia side of the external or 
internal which is the anterior rectus fascia. The transversalis fascia which is adherent to the peritoneum must be identified and opened either by blunt or sharp dissection with care not rupturing the peritoneal sac. At this moment, the operation table is positioned in a Trendelenburg and slightly turned to the contralateral side to facilitate dissection of the peritoneal sac without being disturbed by the viscera.

The preperitoneal space is further developed by using a wet sponge. Instead of the sponge, we can also use the narrow retractors as dissecting instruments. Cleavage begins at the lateral iliac fossa, the peritoneum is dissected free from the lateral and posterior abdominal wall, the iliac vessels must be identified and they are covered by the intact transversalis fascia. Follow these vessels as far as their origin at the iliacus. They are identified by their blue white line with pulsations. The position of these vessels will be used as a reference. The internal ring with the cord structures is automatically localised at its lateral side. An indirect hernia will always be medial anterior to the cord structures. The vessels of the cord are identified by their blue pink colour in lateral dorsal position of the peritoneal sac and ductus deferens by its white structure curving medially and posteriorly. The triangle formed by these structures with very flimsy ligament is the so called "triangle of doom" and lateral from it we find the triangle of pain,[2] the fear of complication during the lateral dissection of the sac in laparoscopic surgery. In this operation both triangles lie directly below the level of grid-iron incision. It makes dissection of the peritoneum which is anterior to the cord much easier and when complications occur they are easily managed by a direct approach. With a Penrose drain, the cord structures can also be isolated and freed as far as cranial at least $10 \mathrm{~cm}$ from the internal ring to facilitate later parietalisation. The thin membrane (urospermatic ligament) connecting the ductus deferens to testicular vessels must be preserved and stay intact during the dissection. If there is a lipoma of the cord which is usually lateral to the cord structures, and if a part of it extends as far as in the inguinal canal it should be drawn from the internal ring and sometimes necessitate removal.

Medial preparation must be continued until the pubic tubercle, Retzius and Bogros' spaces. In primary hernioplasty it will be easy, since most of the peritoneal extrusion medially to the epigastric vessels have already been reduced during the preperitoneal cleavage. To prevent filling in the transversalis envelope after the reduction of a large direct hernia, it is to lateral dorsal of the epigastric vessels and sutured to the anterior abdominal wall. Large peritoneal defects must be closed, preventing the mesh touching the intestines. The femoral area should be examined thoroughly and the presence of a femoral hernia must be excluded. One must carefully reduce the hernia taking care not to injure the femoral vessels.

Complete cleavage of the preperitoneal space can be confirmed by identifying the contralateral pubic tubercle, the Cooper's ligament, the iliac vessels and obturator region medially. The epigastric vessels, internal ring and freed lateral psoas fossa for instruction purposes and for a better view we can use the endoscope to verify the dissected preperitoneal space. Of course, with our finger we can feel the defects and structures in the myopectineal orifice and after mesh placement we can check as well. The posterior lateral part of the iliac fossa, which is narrow must be sufficiently freed from the peritoneal sac. In this part, the mesh must fully incorporate the peritoneal sac, so preventing the recurrence of and indirect hernia. After inspection of all potential hernia sites, the mesh is prepared for the insertion.

A $10 \times 15 \mathrm{~cm}$ polypropylene mesh, the centre of which is marked with a dark coloured suture is trimmed in shape by slightly rounding the corners. The lateral inferior corner is slightly deeper rounded. By doing so, the inferior border will be slightly shorter than the superior border. Using the $30 \mathrm{~cm}$ long anatomy-forceps, the mesh is rolled up in such a way that the intended visceral side of the mesh lies within the roll. We can do this holding up the mesh with visceral side (marked with a coloured stitch), then with the long anatomical forceps the mesh is rolled in its longitudinal axis, starting the roll from the shortest longitudinal border. For the right side, the mesh must be rolled clockwise and the left side anti-clockwise. By doing so, the outer layer of the mesh always opens cranially which makes easier unfurling the cranial part of the mesh.

Meanwhile, the freed preperitoneal space is kept open by three retractors. A retractor of Langenbeck caudomedially protects the epigastric vessels behind it, while the two long narrow retractors hold the preperitoneal sac medially and laterally. The myopectineal orifice, which is meant to be reinforced should be clearly visible. The total length of the rolled mesh is grasped with the long forceps, preventing folding during the insertion. Then the mesh is introduced into the preperitoneal space in the direction of the pubis and must be parallel to the inguinal ligament. The medial end must be behind the pubis, then the centre of the mesh will be medial to the epigastric vessels and the lateral part and border will be at the grid-iron level. Then the lateral superior edge of the mesh is clamped, preventing dislocation during the mesh unfurling manoeuvre. Except the clamp, all retractors are carefully taken out.

Then one of the narrow retractors is reinserted in the centre of the rolled mesh and fixes it against pubis. The second narrow retractor is then carefully inserted below the outer layer and parallel to the first retractor. With a gentle cranial and lateral sweeping movement using the cranial edge of the retractor, the superior part of the prosthesis is unfurled against the anterior abdominal wall.

Then, we use the same technique in a downward direction to unfurl the caudal half of the prosthesis. While the long retractor holds the mid part of the prosthesis against the pubis or abdominal wall, we use a second retractor to unfurl the distal part of the prosthesis. The curves of the lower abdominal wall should be followed during this caudal part unfurling manoeuvre. With the distal end of the retractor, the mesh is unfurled by first streaking out its medial part in Retzius space between bladder and pubis, then in a downward direction covering the Cooper's ligament, femoral until obturator area in the Bogros' space and upward over the iliac and psoas muscle parietalising the cord. The retractor moves in such a way that the peritoneal sac is automatically closed in. The lateral inferior border of the mesh must be laid flat against the abdominal wall, while peritoneal sac resumes its position pushing the prostheses against it. With the two retractors still in place behind the prosthesis, we can inspect either by direct view or using the endoscope. 
Still unfolded parts can be streaked out by using the depressor or the base of forceps. Do not lift the abdomen during the unfurling manoeuvres, because the abdominal wall falls back after it and the mesh will fold in the centre. The mesh should fully retain the peritoneal sac and be placed in such a way that it extends beyond the borders of the myopectineal orifice. When there is any doubt of displacement, we do not hesitate to take the mesh out and start the placement all over again. Care should be taken to protect the epigastric vessels and not to damage it when pulling the mesh out. Since most operations are performed under regional or local anaesthesia the patient could then be asked to cough or strain, testing the integrity of the repair. There will be no bulging at the original hernia side. The interplay of the mesh with the abdominal wall on one side and the peritoneum on the other side is visible. The lateral posterior part of the mesh must fully retain the peritoneal sac and lay flat against the abdomen in the lateral iliac fossa.

One of these sutures fixed is to the lateral anterior part of the mesh for lateral fixation before tissue ingrowths. Do not take a big bite of the mesh, because it will result in folding of the mesh or protruding through the muscle when suture is knotted. We must avoid the ilioinguinal nerve by not taking it in the suture(s). The aponeurosis of the external oblique is also closed with absorbable sutures, after which the skin is closed with interrupted monofilament sutures.

\section{Determination of the Primary Outcome}

The primary outcome of the study was recurrence of hernia within six months after the repair. The patients were followed for a minimum of six months. Post-operatively, each patient was examined for recurrence at discharge, at two weeks, three months and at six months to determine the presence or absence of recurrence. Recurrences were confirmed by examination by another independent surgeon and on doubt by ultrasound examination.

\section{Determination of the Secondary Outcomes}

Secondary outcomes were intraoperative and post-operative complications and patient-centred outcomes. Complications were assessed and recorded intraoperatively and at specified intervals post-operatively. Long-term complications were assessed and documented at three months and six months following discharge from hospital. Patient-centred outcomes (pain, duration of hospital stay and return to work) were assessed at baseline, two weeks, three months and six months. Pain was assessed with the use of a visual-analogue scale at $0,1,4,7,14$ and 28 days post-operatively. Pain was also assessed on the day of discharge for the patients who stayed in the hospital for more than 48 hours after operation. Return to work was assessed by the duration of hospital stay and duration taken to return to previous occupation.

All details of every patient were recorded in individual proforma. The study was designed to detect difference in primary and secondary outcomes between the groups. In the primary analysis, the six-month rates of recurrence were compared between the two groups. The operative time was defined as the time from the first incision to the placement of the last suture.

\section{Complications}

Information about multiple operative and post-operative complications was recorded. The intraoperative complications were problems related to anaesthesia, injury to the vas deferens or peritoneal defect over mesh at closure.

Post-operative complications include early ( $<7$ days) and late ( $>7$ days) complications. Early complications such as urinary retention and urinary tract infection were assessed and documented. Urinary retention was defined as an inability to urinate, requiring catheterisation. A urinary tract infection was recorded only if antibiotic treatment was prescribed. A serious wound infection was defined as the presence of pus or sanguinopurulent discharge at the operative site. Late complications included the same problems as in the early group, but which occurred after 7 days.

The duration of hospital stay as defined by number of days in the hospital after the day of surgery was also recorded. Patients were discharged from the hospital if there was no infection. The patient was able to walk and only oral analgesic therapy was required to manage pain.

The patients were instructed to return to the outpatient clinic at two weeks, three months and at six months for a standardised history taking and physical examination.

The patients were asked to assess the severity of pain at the operative site on the day of operation with the use of a 10 $\mathrm{cm}$ visual analogue scale (scores ranged from 0 for no pain to 10 for unbearable pain) and to record the use of analgesic drugs. Oral analgesia with a non-steroidal anti-inflammatory Diclofenac $50 \mathrm{mg}$ was given on request. Chronic pain was defined as pain in the groin, scrotum or medial part of the thigh that was serious enough for the patient to mention at six months.

The activities of daily living were assessed with a standardised questionnaire. The patients were also asked to record the dates on which they resumed normal daily activity at home and returned to work. All patients were instructed with the same advice about the resumption of work and other activities.

\section{Statistical Method}

Statistical analysis of the duration of operation, duration of hospital stay and resumption of daily activity was done with the help of student's ' $t$ ' test after calculating the arithmetic mean and standard deviation. The pain score was analysed with the help of proportion ' $\mathrm{z}$ ' test after calculating the proportion of patients with each score for each day. The postoperative complications were analysed with odds ratio. All pvalues reported are two sided, and p less than 0.05 denote statistical significance. For statistical analysis, SPSS software version 20 was used.

For the present study, we used statistical software OpenEpi to calculate the sample size assuming recurrence rate of grid-iron technique to be $6.5 \%$ and $2.4 \%$ in Lichtenstein repair, at $95 \%$ confidence level and $80 \%$ power. The estimate sample size of 392 to be achieved in each group for the study is highly improbable to get in our study setting and was also not feasible to get the required sample size in the stipulated period. Statistically, any size $>30$ for each group is considered as large sample, which would facilitate statistical analysis. 


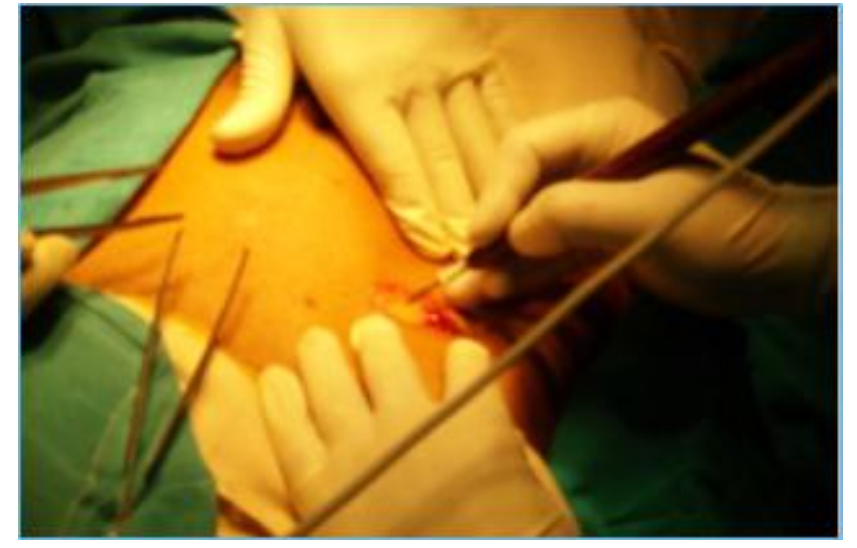

Fig. 1. Incision is Made

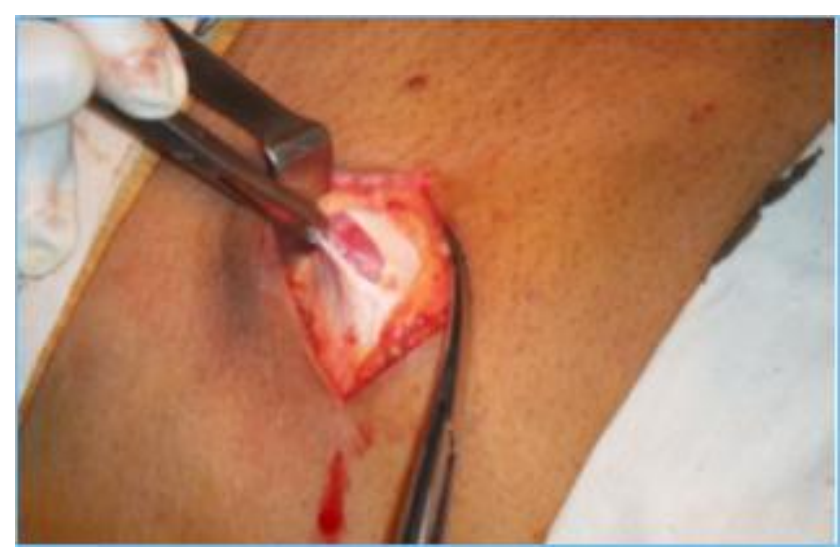

Fig. 2. External Oblique is Split

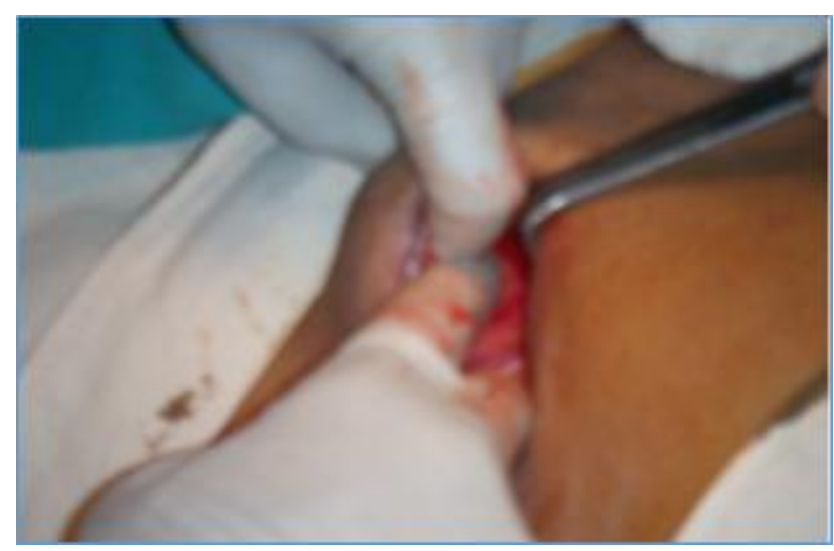

Fig. 3. Preperitoneal Space is Reached

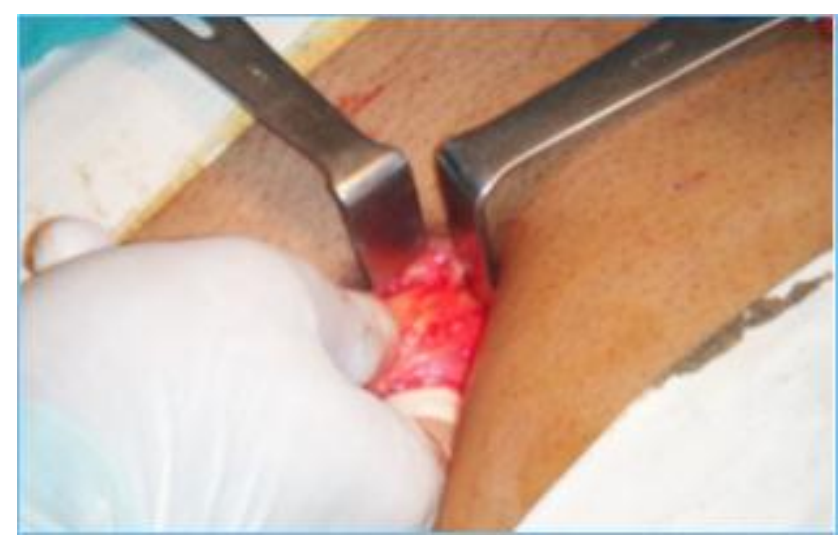

Fig. 4. Sac along with the Cord Structures are Lifted

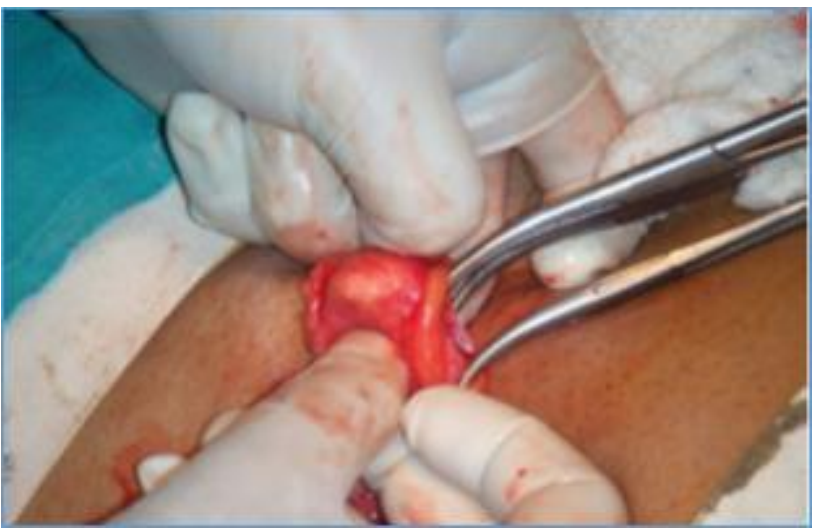

Fig. 5. Sac and Cord are Separated

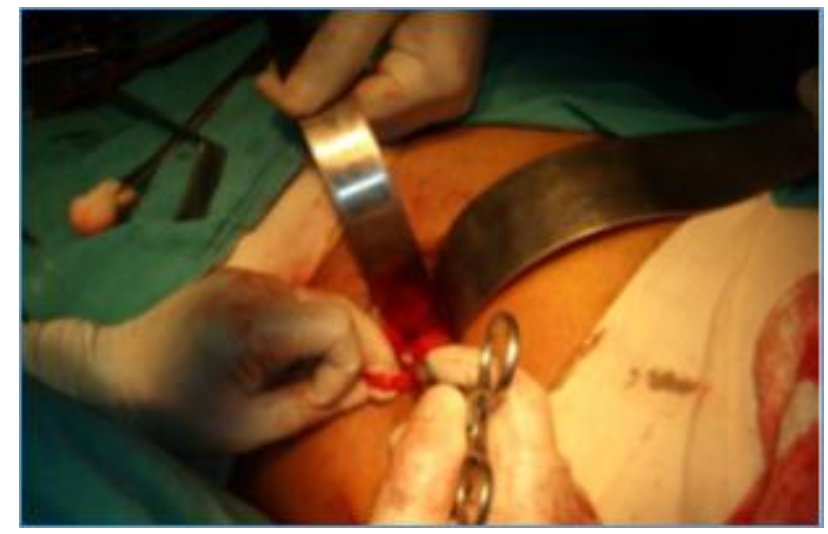

Fig. 6. Placement of Retractors

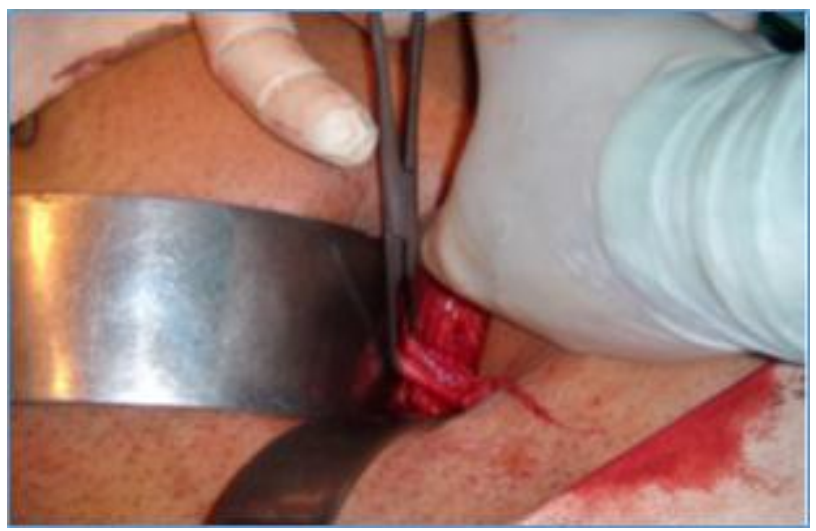

Fig. 7. Parietalisation of the Cord

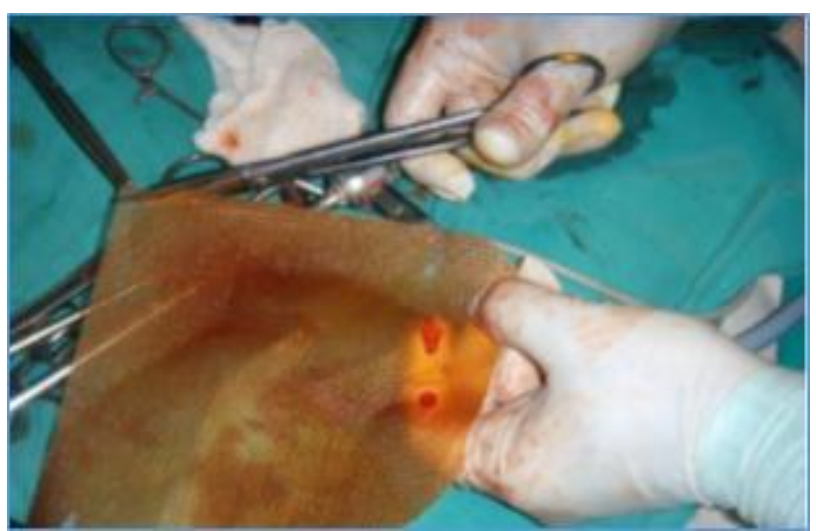

Fig. 8. Mesh is Trimmed 


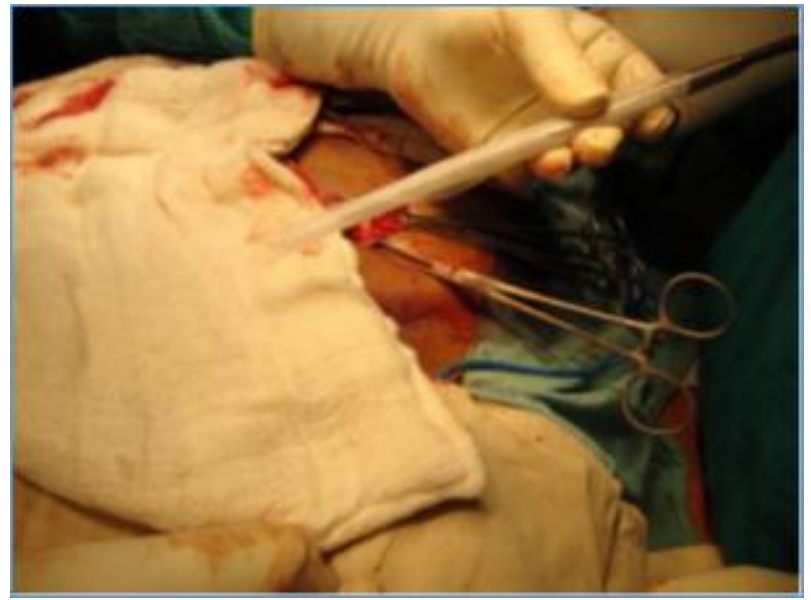

Fig. 9. Mesh is Rolled Up

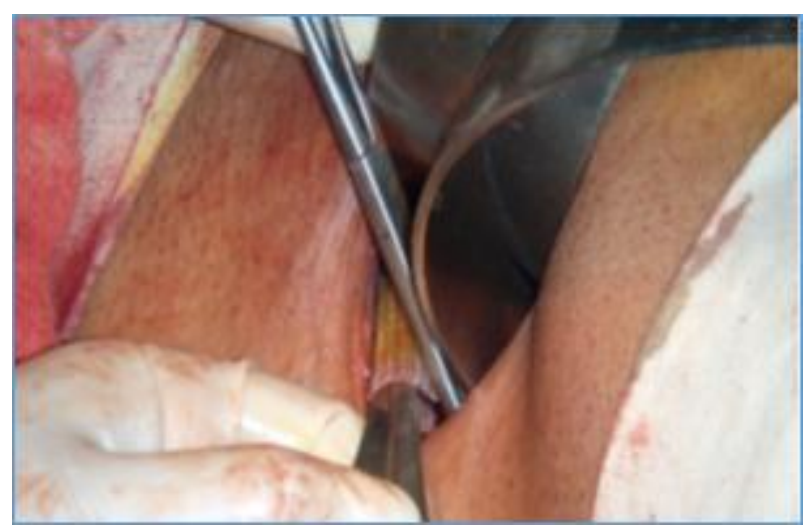

Fig. 10. Placement of the Mesh

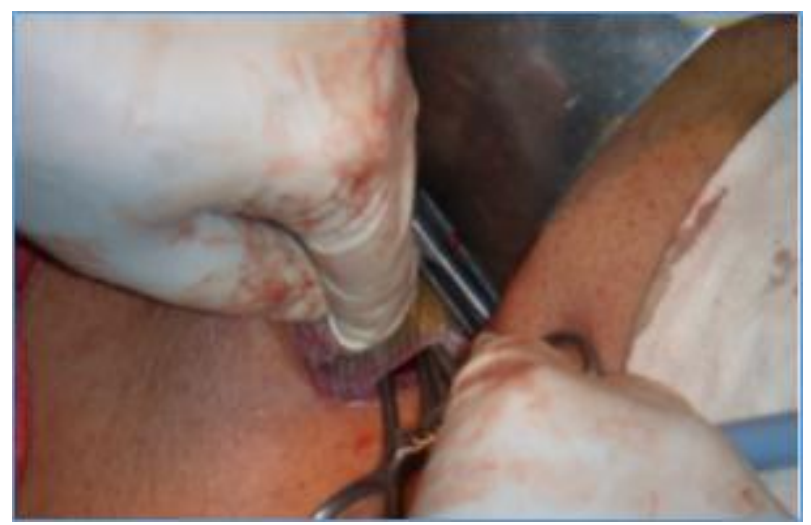

Fig. 11. Mesh is Unfurled

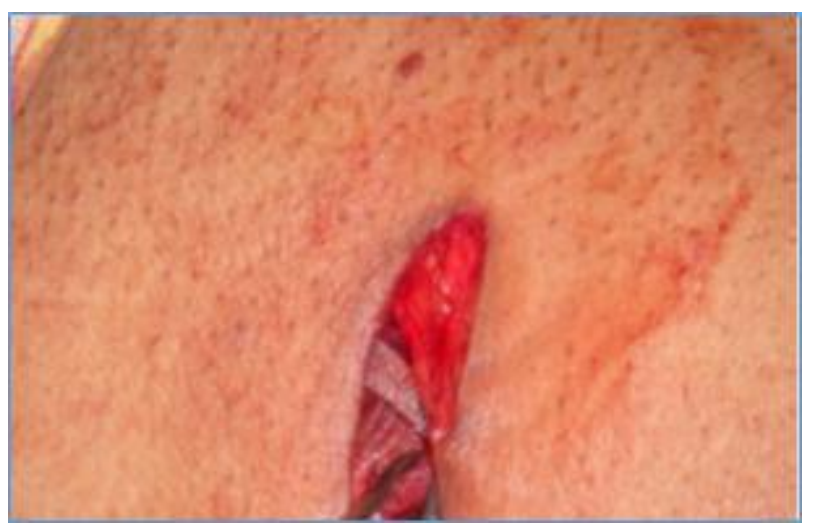

Fig. 12. Mesh is Spread Evenly

\section{RESULTS}

This comparative prospective study was carried out on 60 patients with a clinical diagnosis of inguinal hernia presenting to the Surgery OPD of Medical College and Hospital, Kolkata. They were randomly assigned into two groups for hernia repair, either by Lichtenstein technique or Grid-Iron technique. Thus 30 patients underwent Lichtenstein tension free mesh hernioplasty, whereas 30 patients underwent grid-iron technique of preperitoneal mesh hernioplasty as described by Ugahary.

\section{Patient Characteristics}

The two groups were similar with respect to age, co-morbid condition and type of hernia (Table 1, Table 2 and Table 3 ). In our study, all our patients were male. The two groups were randomly assigned to undergo hernioplasty either by Lichtenstein technique or by Grid-Iron technique.

All patients in both groups were selected after they fulfilled the inclusion/ exclusion criteria as mentioned in materials and methods.

\begin{tabular}{|c|c|c|}
\hline $\begin{array}{c}\text { Age } \\
\text { (Years) }\end{array}$ & $\begin{array}{c}\text { Grid-Iron } \\
\text { Technique }\end{array}$ & $\begin{array}{c}\text { Lichtenstein } \\
\text { Technique }\end{array}$ \\
\hline$<40$ & $10(33.33 \%)$ & $08(26.64 \%)$ \\
\hline $40-60$ & $17(56.61 \%)$ & $18(59.94 \%)$ \\
\hline$>60$ & $03(09.99 \%)$ & $04(13.32 \%)$ \\
\hline \multicolumn{3}{|c|}{ Table 1. Age Distribution of Patients } \\
\hline
\end{tabular}

\section{Type of Hernia}

We selected only those patients who had unilateral hernia only. We excluded the patients with bilateral hernia and those recurrent hernias with more than one operation.

\begin{tabular}{|c|c|c|}
\hline Type of Hernia & $\begin{array}{c}\text { Grid-Iron } \\
\text { Technique }\end{array}$ & $\begin{array}{c}\text { Lichtenstein } \\
\text { Technique }\end{array}$ \\
\hline Primary & $25(83.25 \%)$ & $27(89.91 \%)$ \\
\hline Recurrent & $05(16.65 \%)$ & $03(9.99 \%)$ \\
\hline \multicolumn{2}{|c|}{ Table 2. Type of Hernia } \\
\hline
\end{tabular}

\section{Co-Morbid Conditions}

The co-morbid conditions were properly controlled before going for surgery. The following table shows that our patients had more or less same co-morbid conditions in both the groups.

\begin{tabular}{|c|c|c|}
\hline $\begin{array}{c}\text { Comorbid } \\
\text { Conditions }\end{array}$ & $\begin{array}{c}\text { Grid-Iron } \\
\text { Technique }\end{array}$ & $\begin{array}{c}\text { Lichtenstein } \\
\text { Technique }\end{array}$ \\
\hline Hypertension & $05(16.65 \%)$ & $03(09.99 \%)$ \\
\hline COPD & $04(13.32 \%)$ & $02(06.66 \%)$ \\
\hline Prostatism & $01(03.33 \%)$ & $02(06.66 \%)$ \\
\hline Diabetes & $03(09.99 \%)$ & $01(03.33 \%)$ \\
\hline Smoking & $07(23.31 \%)$ & $05(16.65 \%)$ \\
\hline \multicolumn{2}{|c|}{ Table 3. Co-morbid Condition } \\
\hline
\end{tabular}

\section{Duration of Operation}

\begin{tabular}{|c|c|c|c|}
\hline $\begin{array}{c}\text { Type of } \\
\text { Hernia }\end{array}$ & $\begin{array}{c}\text { Grid-Iron } \\
\text { Technique } \\
\text { a.m }+/- \text { s.d }\end{array}$ & $\begin{array}{c}\text { Lichtenstein } \\
\text { Technique } \\
\text { a.m }+/- \text { s.d }\end{array}$ & P value \\
\hline Primary & $74+/-8(\mathrm{n}=25)$ & $34+/-6(\mathrm{n}=27)$ & $<.0001$ \\
\hline Recurrent & $60+/-5(\mathrm{n}=5)$ & $70+/-23(\mathrm{n}=3)$ & $<.362$ \\
\hline \multicolumn{4}{|c|}{ Table 4. Duration of Operation (Avg. Minutes) in Two } \\
Groups
\end{tabular}


The above table shows that the mean time for primary hernia repair by grid-iron technique was more than the Lichtenstein technique. This value was statistically significant ( $\mathrm{p}$ value $<.01$ ). We calculated this by two-tailed student ' $\mathrm{t}$ ' test. However, the operative time for the recurrent hernia repair had no difference.

\section{Post-Operative Pain}

Post-operative pain was assessed by visual analogue scale (VAS) (0- no pain, 10- unbearable pain) at day $0,1,4,7,14$ and 28 after the operation. To quantify the nature of pain the patient experienced during the whole course of either of the hernioplasty procedures and during the post-operative follow-up period, we simplified the pain score data without modifying the original scoring system for the better interpretation of the results which can be extrapolated to the greater population of the patients. This is as follows:
i. A means pain score between 0-1
ii. B means pain score between 1-2
iii. C means pain score between 2-4
iv. D means pain score between 4-6
v. E means pain score between 6-8
vi. F means pain score between 8-9
vii. G means pain score between 9-10

\begin{tabular}{|c|c|c|c|c|c|c|c|c|}
\hline $\begin{array}{c}\text { Pain Grade } \\
\text { Day }\end{array}$ & A & B & C & D & E & F & G & Total \\
\hline 0 & & 11 & 16 & 03 & & & & 30 \\
\hline 1 & & 27 & 03 & & & & & 30 \\
\hline 4 & 30 & & & & & & & 30 \\
\hline 7 & 30 & & & & & & & 30 \\
\hline 14 & 30 & & & & & & & 30 \\
\hline 28 & 30 & & & & & 30 \\
\hline Iron Hernioplasty \\
\hline Table 5a. Grading of Patients according to VAS after Grid- \\
\hline
\end{tabular}

\begin{tabular}{|c|c|c|c|c|c|c|c|c|}
\hline $\begin{array}{c}\text { Pain Grade } \\
\text { Day }\end{array}$ & A & B & C & D & E & F & G & Total \\
\hline 0 & & 03 & 06 & 21 & & & & 30 \\
\hline 1 & & 08 & 22 & & & & & 30 \\
\hline 4 & & 10 & 20 & & & & & 30 \\
\hline 7 & & 22 & 08 & & & & & 30 \\
\hline 14 & & 30 & & & & & & 30 \\
\hline 28 & 30 & & & & & & & 30 \\
\hline
\end{tabular}

\begin{tabular}{|c|c|c|c|c|}
\hline DAY & vas & G-I TECH & LTECH & $\mathrm{P}$ value \\
\hline \multirow[t]{4}{*}{0} & $A$ & & & \multirow[t]{4}{*}{$<.01$} \\
\hline & $B$ & $11 / 30$ & $03 / 30$ & \\
\hline & $C$ & $16 / 30$ & $06 / 30$ & \\
\hline & $\mathrm{D}$ & $03 / 30$ & $21 / 30$ & \\
\hline \multirow[t]{4}{*}{1} & $\mathrm{~A}$ & & & \multirow[t]{4}{*}{$<.01$} \\
\hline & $B$ & $27 / 30$ & $08 / 30$ & \\
\hline & C & $03 / 30$ & $22 / 30$ & \\
\hline & D & & & \\
\hline \multirow[t]{4}{*}{4} & $\mathrm{~A}$ & $30 / 30$ & & \\
\hline & $B$ & & $10 / 30$ & \\
\hline & $C$ & & $20 / 30$ & \\
\hline & D & & & \\
\hline \multirow[t]{3}{*}{7} & A & $30 / 30$ & & \\
\hline & $B$ & & $22 / 30$ & \\
\hline & C & & $08 / 30$ & \\
\hline
\end{tabular}

Table 5c.

\begin{tabular}{|l|l|l|l|l|}
\hline & D & & & \\
\hline \multirow{4}{*}{14} & A & $30 / 30$ & & \\
\cline { 2 - 4 } & B & & $30 / 30$ & \\
\cline { 2 - 4 } & C & & & \\
\cline { 2 - 4 } & D & & & \\
\hline
\end{tabular}

\begin{tabular}{|l|l|l|l|l|}
\hline \multirow{5}{*}{28} & $\mathrm{~A}$ & $30 / 30$ & & \\
& $\mathrm{~B}$ & $30 / 30$ & & \\
\hline & $\mathrm{C}$ & & & \\
\hline & $\mathrm{D}$ & & & \\
\hline
\end{tabular}

After comparing the above table, we can see that the maximum intensity of pain experienced by the two groups was D on the day of operation and this was reported by only 3 patients who underwent grid-iron repair, while 21 patients reported in the other group. However, on 28th post-op day both the groups had similar experience of pain. The statistical value was calculated by proportion ' $\mathrm{z}$ ' test, which showed that there was statistical significance on day $0,1,4,7$ and 14, but not on the day 28 .

\section{Post-Operative Complications}

\begin{tabular}{|l|l|l|}
\hline VARIABLE & $\begin{array}{l}\text { GRID-IRON } \\
\text { TECHNIQUE }\end{array}$ & $\begin{array}{l}\text { LICHTENSTEIN } \\
\text { TECHNIQUE }\end{array}$ \\
\hline RELATED TO ANAESTHESIA & 0 & 0 \\
\hline INJURY TO VESSELS & 1 & 0 \\
\hline $\begin{array}{l}\text { INJURY TO CORD } \\
\text { STRUCTURES }\end{array}$ & 2 & 1 \\
\hline PERITONEAL INJURY & 1 & 0 \\
\hline URINARY RETENTION & 1 & 2 \\
\hline URINARY TRACT INFECTION & 0 & 0 \\
\hline HEMATOMA/SEROMA & 3 & 4 \\
\hline ORCHITIS & 2 & 1 \\
\hline WOUND INFECTION & 0 & 1 \\
\hline
\end{tabular}

\section{Table 6. Post-Operative Complications}

The above table shows that peritoneal injury was significant in grid-iron technique; otherwise, the other complications were almost same in both the groups. On calculating the odds ratio of the complications of the two procedures as shown in the table below it is not significant.

Odds Ratio of the Complications between the Two Groups Odds ratio: $10 \times 21 / 09$ × $20=1.16$

The gird-iron technique has 1.16 times more complications than the Lichtenstein method.

\section{Recurrence}

Patients were followed up for 6 months for the recurrence; 2 $(6.67 \%)$ recurrences occurred in patients who underwent Grid-Iron technique of repair.

\section{Duration of Hospital Stay}

\begin{tabular}{|l|l|l|l|}
\hline & $\begin{array}{l}\text { GRID-IRON } \\
\text { TECHNIQUE } \\
\text { a.m+/-s.d }\end{array}$ & $\begin{array}{l}\text { LICHTENSTEIN } \\
\text { TECHNIQUE } \\
\text { a.m+/-s.d }\end{array}$ & p value \\
\hline $\begin{array}{l}\text { STAY IN HOSPITAL } \\
\text { (DAYS) }\end{array}$ & $2+/-0.8(n=30)$ & $2.7+/-1 \quad(n=30)$ & $<.014$ \\
\hline
\end{tabular}

\section{Table 7. Duration of Hospital Stay}

After both the operations, patients were discharged when they can urinate and walk without postural hypotension. The patient leaves with analgesics and instructions. They were enquired over the phone about their progress. The above 
table shows that those treated with grid-iron technique had shorter duration of hospital stay.

\section{Resumption to Daily Activity}

Patients were asked to note how many days after the operation they resumed their daily activities. These were noted for the two groups.

\begin{tabular}{|l|l|l|l|}
\hline & $\begin{array}{l}\text { GRID-IRON } \\
\text { TECHNIQUE } \\
\text { a.m+/-s.d }\end{array}$ & $\begin{array}{l}\text { LICHTENSTEIN } \\
\text { TECHNIQUE } \\
\text { a.m+/-s.d }\end{array}$ & p value \\
\hline RESUMPTION TO & $3+/-0.9 \quad(\mathrm{n}=30)$ & $7+/-1 \quad(\mathrm{n}=30)$ & $<0.0001$ \\
\hline $\begin{array}{l}\text { DAILY ACTIVITY } \\
\text { (DAYS) }\end{array}$ & & & \\
\hline
\end{tabular}

The above table shows that the patients treated with gridiron technique returned to their daily activity early compared to Lichtenstein technique.

\section{DISCUSSION}

The distribution of patients in the two groups was similar. In both groups, the higher grades of hernias were not present. This has probably arisen from the selection bias, as we wanted to compare the results among the more common categories of hernias.

In our study, the average operating time was 74 and 60 minutes for primary and recurrent hernias respectively by grid-iron technique. It took 30 and 70 minutes for primary and recurrent hernias respectively by Lichtenstein method. So there was statistical significance in case of primary hernia repair, where the grid-iron technique took significantly more time than the Lichtenstein procedure. The increased operating time points to the difficulties faced, while performing the grid-iron technique due to slow learning curve. It is interesting to note that the time taken for repair of recurrent hernias was almost same for both the procedures. But it was not statistically significant.

Proportional odds modelling of visual pain scores at 0,1 , 4, 7 and 14 days after surgery showed significantly less postoperative pain for grid iron technique than the Lichtenstein technique. Post-operatively, patients indicated their levels of pain at various time intervals by using VAS (visual analogue scale). However, there was no statistical significance in the pain scores between the two groups at 28 days.

There were minor complications in both the procedures post-operatively. In grid-iron method 10 patients (33.33\%) developed minor complications related to operation such as haematoma, seroma and retention of urine. One patient had peritoneal injury during operation, which was managed peroperatively through the same incision. The odds ratio between the two techniques shows that the grid-iron technique was 1.16 times than the Lichtenstein technique with relation to the post-operative complications.

\section{Technical Problems in the Grid-Iron Repair}

a) Difficulty in identifying the fascia transversalis, the tiny membrane before the peritoneum.

b) Accidentally, the epigastric vessels may be freed from this fascia attachment and stripped, which causes bleeding. c) Insufficient freeing of the sac from the cord may result in a complete incorporation of the sac within the mesh and may result in recurrence of the indirect hernia.

d) Before the insertion of the mesh, the retractors are to be placed properly to expose the MPO.

e) Bladder must be catheterised beforehand to avoid injury during operation.

Overall, recurrence rates were higher among patients whose hernias were repaired by the grid-iron technique, possibly due to the long learning curve of the technique. There were 2 recurrences among 30 patients [6.67\%] than none after Lichtenstein method of hernia repair.

The duration of hospital stay was significantly lower in the grid-iron group compared to the Lichtenstein group $(p<0.014)$. The mean duration of post-operative hospital stay in the grid-iron method is 2.1 days as compared to 2.7 in the Lichtenstein group.

The patients who underwent grid-iron repair returned to their usual activities by $3-4$ days sooner than those who underwent repair by Lichtenstein method (mean time, 7 days).

\section{CONCLUSION}

Lichtenstein repair of inguinal hernia is a time-tested method of treatment of inguinal hernia. It is associated with the lowest rate of recurrence among the various methods of repair. Being tension-free, it allows the patient to early return to previous state of activity. The Lichtenstein technique is easier to learn and easy to implement for the average general surgeon.

On the other hand, grid-iron technique developed by Ugahary is a new method of inguinal hernia repair where the mesh is placed in the preperitoneal space. It is considered a more physiological repair having all the advantages of minimal access surgery like of quicker return to work, less post-operative pain and a better cosmetic result. The recurrence rate is due to difficulty in reproducing the procedure in non-expert hands. The longer duration of operation is also due to the long learning curve and difficulty in execution of the technique. A good knowledge of anatomy as well as intensive training and proper experience is essential to execute this technique. The period of study is inadequate to evaluate the outcome of this technique with respect to recurrence. Longer periods of followup are required to comment on this aspect. However, the results of study are encouraging enough to conclude that the technique followed is a novel, new and rational method of treating groin hernias.

\section{REFERENCES}

[1] Wantz GE. Abdominal wall hernias. In: Schwartz (eds). Principles of surgery. $8^{\text {th }}$ edn. Mcgraw-Hill healthy professions division, 1999;13:53.

[2] Bassini E. Nouvo metodo operative per la cura radicale dell' ernia inguinale. Padua, Italy: Prosperini; 1889;23:13.

[3] Abramson JH, Gofin J, Hopp C, et al. The epidemiology of inguinal hernia. A survey in western Jerusalem. J Epidemiol Community Health 1978;32(1):59-67. 


\section{Jemds.com}

[4] Akin ML, Karakaya M, Batkin A, et al. Prevalence of inguinal hernia in otherwise healthy males of 20 to 22 years of age. J R Army Med Corps 1997;143(2):101-2.

[5] Shouldice EE. The treatment of hernia. Ontario Med Rev 1953;20:670-84.

[6] Lichtenstein IL, Schulman AG. Ambulatory outpatient hernia surgery. Including a new concept, introducing tension-free repair. Int Surg 1986;71(1):1-4.

\section{Original Research Article}

[7] Ugahary F, Simmermacher RKJ. Groin hernia repair via a grid-iron incision: an alternative technique for preperitoneal mesh insertion. Hernia 1998;2(3):1235.

[8] Ugahary F. The grid-iron hernioplasty. Bendavid R, Abrahamson J, Arregui ME, et al. (eds). Abdominal Wall hernias. Principles and management. Springer Verlag, New York, Berlin Heidelberg, 2001;59:407-11. 